\title{
Review Article \\ Scientific Evaluation of Edible Fruits and Spices Used for the Treatment of Peptic Ulcer in Traditional Iranian Medicine
}

\author{
Mohammad Hosein Farzaei, ${ }^{1}$ Mohammad Reza Shams-Ardekani, ${ }^{1,2}$ \\ Zahra Abbasabadi, ${ }^{3}$ and Roja Rahimi ${ }^{1}$ \\ ${ }^{1}$ Department of Traditional Pharmacy, Faculty of Traditional Medicine, Tehran University of Medical Sciences, \\ Tehran 1417653761, Iran \\ ${ }^{2}$ Department of Pharmacognosy, Faculty of Pharmacy, Tehran University of Medical Sciences, Tehran 1417614411, Iran \\ ${ }^{3}$ Faculty of Pharmacy, Kermanshah University of Medical Sciences, Kermanshah 6734667149, Iran
}

Correspondence should be addressed to Roja Rahimi; rojarahimi@gmail.com

Received 26 June 2013; Accepted 24 July 2013

Academic Editors: J. M. Pajares, R. G. Romanelli, and W. Vogel

Copyright (C) 2013 Mohammad Hosein Farzaei et al. This is an open access article distributed under the Creative Commons Attribution License, which permits unrestricted use, distribution, and reproduction in any medium, provided the original work is properly cited.

\begin{abstract}
In traditional Iranian medicine (TIM), several edible fruits and spices are thought to have protective and healing effects on peptic ulcer (PU). The present study was conducted to verify anti-PU activity of these remedies. For this purpose, edible fruits and spices proposed for the management of PU in TIM were collected from TIM sources, and they were searched in modern medical databases to find studies that confirmed their efficacy. Findings from modern investigations support the claims of TIM about the efficacy of many fruits and spices in PU. The fruit of Phyllanthus emblica as a beneficial remedy for PU in TIM has been demonstrated to have antioxidant, wound healing, angiogenic, anti-H. pylori, cytoprotective, antisecretory, and anti-inflammatory properties. The fruit of Vitis vinifera has been found to be anti-H. pylori, anti-inflammatory, wound healing, angiogenic, cytoprotective, and antioxidant. The fruit and aril of seed from Myristica fragrans exert their beneficial effects in PU by increasing prostaglandin, modulation of nitric oxide and inflammatory mediators, wound healing, antisecretory, antacid, antioxidant, and anti- $H$. pylori activities, and improving angiogenesis. Pharmacological and clinical studies for evaluation of efficacy of all TIM fruits and spices in PU and their possible mechanisms of action are recommended.
\end{abstract}

\section{Introduction}

Gastric and duodenal ulcers, entitled as peptic ulcer (PU), are the most prevalent gastrointestinal disorders in the world [1]. PU is a multifactorial and complex disease with unclear etiological factor. It has been demonstrated that $\mathrm{PU}$ is a pathological condition in which biological balance between aggressive and defense factors is disturbed. Among aggressive factors, it can be named from gastric acid and pepsin secretion, active free radicals and oxidants, leukotrienes, endothelins, and exogenous factors such as ethanol or nonsteroidal anti-inflammatory drugs (NSAIDs). In contrast, gastric mucus, bicarbonate, normal blood flow, prostaglandin $(\mathrm{PG})$, nitric oxide (NO), and antioxidant enzymes such as catalase and glutathione (GSH) work as defense factors $[2,3]$.
Most of the gastric lesions originate from a chronic infection of gastric mucosa with Helicobacter pylori (H. pylori). $H$. pylori is a common human pathogen with asymptomatic stomach colonization in nearly $70 \%$ of the population and approximately $10 \%-20 \%$ are susceptible for PU [4].

Traditional medicines of all over the world possess different virgin remedies for the treatment of symptomatologies related to many ailments. Thus, they are very important for investigation on their efficacy and phytochemical constituents [5-7]. There are several edible fruits and spices proposed in traditional Iranian medicine (TIM) for the management of PU $[8,9]$. Present study conducted to review these fruits and spices and found evidence for their efficacy and biological mechanisms in modern publications. In order to achieve this aim, electronic databases including PubMed, 
Scopus, Web of Science, and Google Scholar were explored for each of the medicinal plants recommended in TIM for PU, and all retrieved articles were evaluated to obtain any in vitro, in vivo, or clinical evidence for their efficacy and possible mechanisms. The retrieved studies either explain clearly effectiveness of these herbs or indirectly their efficacy on the involved mechanisms in the treatment of PU.

\section{Edible Fruits and Spices for the Treatment of PU in TIM}

Scientific, common English and traditional Iranian names of edible fruits and spices used in TIM for the management of PU with their plant family and pharmacological activities in TIM have been shown in Table 1. Moreover, details of in vitro and in vivo findings that support their efficacy in PU have been demonstrated in Table 2. Below, these fruits and spices with their possible mechanisms of action in PU have been described alphabetically.

2.1. Amygdalus communis. A. communis demonstrated a trivial antacid property in vitro [10], and different parts of fruit showed antioxidant activity [11, 12]. Topical application of bitter almond oil healed wounds in rats [13]. Amygdalin, a glycoside isolated from Amygdalus genus, revealed gastroprotective properties through suppression of inflammatory cytokines [14].

2.2. Berberis vulgaris $L$. Fruit has shown antioxidant activity [15] and may have a role in improvement of intestinal mucosal morphology [18]. Berberine, as an active constituent of fruit, promoted releasing $\mathrm{NO}$ in the intestinal endothelium [16]. It showed inhibitory activity on $H$. pylori growth [17] and gastroprotective effect through modulating NO synthase (NOS) gene expression [19]. It also had protective activity against small intestinal injury and increased adenosine in the intestinal tissue [20]. However, there is a report about dose-dependent gastric ulcer inducing activity of berberine from $B$. crataegina during acute toxicity test in mice [21].

2.3. Cornus mas $L$. The fruits showed antioxidant activity [22]. The leaves of C. controversa, C. macrophylla, and C. walteri demonstrated anti-H. pylori activity [23].

2.4. Cucurbita maxima Duch. and C. Pepo L. Fruit pulp of C. pepo showed protective activity against gastric and duodenal ulcer via enhancing mucosal thickness and increasing alkaline phosphatase enzyme in stomach and duodenum tissue [25]. Triterpenoids from the seeds of C. pepo protected against gastric ulcer via reducing gastric secretion and free and total acidity of gastric juice and its antioxidant activity [24].

2.5. Cydonia vulgaris Pers. syn. C. oblonga Mill. Various components from peel, pulp, and seed of fruit exhibited antioxidant activity [26]. Phenolic compounds from fruits showed gastroprotective properties [27]. Fruit juice and fruit extract demonstrated strong and weak anti- $H$. pylori activity, respectively [29]. The seed mucilage topically administrated heals toxin-induced skin lesions in rabbits [28].

2.6. Malus domestica Baumg. M. domestica fruit and its isolated phenolic acids demonstrated gastroprotective activity via reducing neutrophil infiltration in gastric tissue and antioxidant activity [30,31]. The fruit also reduced gastric endothelial cell injury through antioxidant activity [31]. $M$. domestica peel showed both in vitro and in vivo anti $H$. pylori activity $[32,33]$. Fruit polyphenol revealed gastroprotective activity without significant effect on gastric secretion. It also inhibited lipid peroxidation and production of inflammatory cytokines [34]. However, there is a report on exacerbation of gastric ulcer by fruit polyphenol extract [27].

2.7. Morus alba L. and M. nigra L. Cyanidin-3-O-glucoside, a component isolated from $M$. alba fruit, showed protective activity against endothelial dysfunction [36]. Ethyl acetate soluble fraction of fruit attenuated gastric ulceration in rat via its antioxidant activity [38]. The leaf had protective activity against gastric ulcer [35] and revealed anti $H$. pylori as well as antioxidant activity [37].

2.8. Myristica fragrans Houtt. Various investigations have proved strong anti-H. Pylori activity of $M$. fragrans seed in vitro [39]. Dihydroguaiaretic acid isolated from aril of the seed also demonstrated strong anti H. pylori activity [40]. The seeds suppressed free and total acidity and volume of gastric secretion [42]. The aril of seed showed antioxidant activity in vitro [41]. M. malabarica fruits improved gastric ulcer in mice via increasing PG E2 synthesis, improving angiogenesis, modulating NOS gene expression, producing balance between proinflammatory and anti-inflammatory cytokines, and improving mucin content and antioxidant activity in gastric tissue [44-46]. M. andamanica leaves demonstrated wound healing activity in vivo [47]. A polyherbal formulation containing $M$. fragrans fruits inhibits gastric ulcer and hypersecretion in rats [43].

2.9. Oryza sativa $L$. O. sativa bran oil protected gastric mucus from stress-induced ulcers in rats via inhibiting acid secretion. $O$. sativa reduced basal acid secretion and stimulated gastric acid secretion by histamine in rats [50]. Antioxidant activity of normal and pigmented rice brans and some isolated components has been proved in vitro [48]. $O$. sativa cooked seeds suppressed intestinal secretion through inhibiting the response of intestinal epithelial crypt cells to adenosine $3^{\prime}, 5^{\prime}$-cyclic monophosphate, a major intracellular mediator of secretion [51]. Rice fluid exhibited strong bactericidal activity against $H$. pylori [49]. In spite of these supportive data, Jayaraj et al. demonstrated that oil derived from rice and rice bran on storage becomes ulcerogenic, while fresh rice bran diet protected mucosa from ulceration [52]. The study evaluating dietary profile of patients with duodenal ulcer showed more ulcer occurrence in patient with rice diets. 
TABLE 1: Medicinal plants used for the treatment of peptic ulcer in traditional Iranian medicine $[8,9]$.

\begin{tabular}{|c|c|c|c|c|}
\hline Scientific names & Family & $\begin{array}{c}\text { Common } \\
\text { English name }\end{array}$ & $\begin{array}{l}\text { Name(s) in TIM } \\
\text { resources }\end{array}$ & Uses in TIM \\
\hline Amygdalus communis & Rosaceae & Almond & Badam, Lowz & $\begin{array}{l}\text { Respiratory disorders, brain } \\
\text { tonic, and PU }\end{array}$ \\
\hline Berberis vulgaris & Berberidaceae & $\begin{array}{l}\text { Common } \\
\text { barberry }\end{array}$ & $\begin{array}{c}\text { Zereshk, } \\
\text { Ambarbaris, and } \\
\text { Arghis (root) }\end{array}$ & $\begin{array}{l}\text { Gastric tonic, liver disease, } \\
\text { dyspepsia, and PU }\end{array}$ \\
\hline Cornus mas & Cornaceae & $\begin{array}{l}\text { Cornelian } \\
\text { cherry }\end{array}$ & Zoghal, Zoghal akhte & Gastritis, hepatitis, IBD, and PU \\
\hline Cucurbita maxima, C. pepo & Cucurbitaceae & Pumpkin & Kadou, Ghar & Wound healer, and PU \\
\hline Cydonia vulgaris & Rosaceae & Quince & Safarjal, Beh & $\begin{array}{c}\text { Antidepressant, gastralgia, and } \\
\text { PU }\end{array}$ \\
\hline Malus domestica & Rosaceae & Apple & Sib, Toffah & $\begin{array}{c}\text { Antidepressant, dysentery, and } \\
\text { PU }\end{array}$ \\
\hline Morus alba, $M$. nigra & Moraceae & $\begin{array}{l}\text { White and } \\
\text { black } \\
\text { mulberry }\end{array}$ & Tout sefid, Tout siah & $\begin{array}{l}\text { Liver and spleen disorders, } \\
\text { aphrodisiac, diuretic, and PU }\end{array}$ \\
\hline Myristica fragrans & Myristicaceae & $\begin{array}{l}\text { Nutmeg, mace } \\
\text { (aril of seed) }\end{array}$ & $\begin{array}{l}\text { Jowz bouya (fruit), } \\
\text { Basbase (aril of seed) }\end{array}$ & $\begin{array}{c}\text { Gastric and liver tonic, PU, and } \\
\text { aphrodisiac }\end{array}$ \\
\hline Oryza sativa & Gramineae & Rice & Oroz, Berenj & IBD, PU, and aphrodisiac \\
\hline Phoenix dactylifera & Arecaceae & Date & Khorma & $\begin{array}{l}\text { Antidepressant, wound healer, } \\
\text { aphthous, and PU }\end{array}$ \\
\hline Phyllanthus emblica & Phyllanthaceae & Gooseberry & Amole & $\begin{array}{c}\text { Memory enhancer, appetizer, } \\
\text { and PU }\end{array}$ \\
\hline Punica granatum & Punicaceae & Pomegranate & Anar, Roman & $\begin{array}{l}\text { Gastric and liver tonic, PU, and } \\
\text { IBD }\end{array}$ \\
\hline Rhus coriaria & Anacardiaceae & Sumac & Sumac & $\begin{array}{l}\text { Gastric tonic, appetizer, } \mathrm{PU} \text {, and } \\
\text { hemorrhage }\end{array}$ \\
\hline Vitis vinifera & Vitaceae & Grape & $\begin{array}{l}\text { Mow (tree), Ghoureh } \\
\text { (unripe fruit), and } \\
\text { Angour (ripe fruit) }\end{array}$ & Wound healer, hematopoietic \\
\hline
\end{tabular}

TIM: traditional Iranian medicine, PU: peptic ulcer, and IBD: inflammatory bowel disease.

Moreover, mucin activity was attenuated, and severity of ulcer induced by pylorus ligation was higher in rice diet rats [53].

2.10. Phoenix dactylifera L. Fruit and seed possess antioxidant activity $[54,55]$. The fruit ameliorated gastric ulcers via increasing gastric mucin and reducing histamine and gastrin (a gastrointestinal hormone that regulates gastric acid secretion, releases histamine, and regulates gastric endocrine cell proliferation in the plasma) [56].

2.11. Phyllanthus emblica L. P. emblica fruit purified phenolics demonstrated antioxidant activity in vitro [57]. The fruit exhibited wound healing activity via improvement of collagen function and enhancing antioxidant capacity [59]. It protected against gastric ulcer via its antioxidant and cytoprotective activity $[60,62]$. Gallic acid enriched extract exhibited healing property on gastric ulcer via increasing $P G E_{2}$ and proangiogenesis factors, enhancing endothelial NOS (eNOS), and regulation of pro-inflammatory and antiinflammatory cytokines and antioxidant activity $[61,63]$. The fruit ethanol extract demonstrated anti $H$. pylori activity in vitro [58].

2.12. Punica granatum L. P. granatum peel extract protected gastric mucus from gastric ulcer via its antioxidant activity and attenuating gastric acidity [67, 68]. It attenuated acetylcholine-induced contractions and inhibition of the spontaneous movement of the isolated rat ileum [64]. The peel also showed anti $H$. pylori activity [65]. The ointment prepared from the peel extract accelerated wound healing and exhibited antioxidant properties in guinea pigs [66]. The tannins from fruit prevented formation of gastric ulcer, increased NO level and secretion of adherent and free mucus, and exhibited antioxidant activity in gastric mucosa [69].

2.13. Rhus coriaria $L$. The fruit demonstrated antioxidant activity in vitro [71, 72]. Ethanol extract of fruit showed antibacterial activity against $H$. pylori $[70]$.

2.14. Vitis vinifera $L$. The seed demonstrated antioxidant activity in vitro [73]. The fruit skin and seed revealed anti 
TABLE 2: Pharmacological activities attributed to antipeptic ulcer activity of edible fruits and spices used in TIM for the management of this disease.

\begin{tabular}{|c|c|c|c|c|c|c|}
\hline Plant & Part/extract & Active constituent & Model & Species & Result & Reference \\
\hline \multirow{5}{*}{$\begin{array}{l}\text { Amygdalus } \\
\text { communis }\end{array}$} & Powdered fruit & - & In vitro & - & Antacid & {$[10]$} \\
\hline & $\begin{array}{l}\text { Hull and shell/methanol } \\
\text { extract }\end{array}$ & - & In vitro & - & Antioxidant & [11] \\
\hline & $\begin{array}{l}\text { Defatted seed } / 80 \% \\
\text { acetone extract and its } \\
\text { fractions }\end{array}$ & - & In vitro & - & Antioxidant & [12] \\
\hline & Nut/oil & - & Open wound & Rat & Wound healing & {$[13]$} \\
\hline & - & Amygdalin & Ethanol-induced GU & Rat & $\begin{array}{l}\downarrow G U \text {, gastric secretion } \\
\text { and inflammatory } \\
\text { agents: TNF- } \alpha \text { and NO }\end{array}$ & [14] \\
\hline \multirow{7}{*}{ Berberis vulgaris } & $\begin{array}{l}\text { Fruit/ethanol, methanol } \\
\text { and water extract }\end{array}$ & - & In vitro & - & Antioxidant & {$[15]$} \\
\hline & - & Berberine & In vitro & - & $\begin{array}{l}\uparrow N O \text { in intestinal } \\
\text { endothelium cell }\end{array}$ & [16] \\
\hline & - & Berberine & In vitro & - & Anti-H. pylori & [17] \\
\hline & Fruit/water extract & - & - & $\begin{array}{l}\text { Broiler } \\
\text { chicken }\end{array}$ & $\begin{array}{l}\text { Improvement of } \\
\text { intestinal mucosal } \\
\text { morphology }\end{array}$ & {$[18]$} \\
\hline & - & Berberine & Ethanol-induced GU & Mouse & $\begin{array}{l}\downarrow \mathrm{GU}, \uparrow \mathrm{eNOS} \text {, and } \\
\text { } \mathrm{iNOS} \text { mRNA } \\
\text { expressions }\end{array}$ & [19] \\
\hline & - & Berberine & $\begin{array}{l}\text { Indomethacin-induced } \\
\text { small intestinal injury }\end{array}$ & Mouse & $\begin{array}{l}\downarrow \text { Intestinal injury, } \\
\uparrow \text { adenosine of intestinal } \\
\text { tissue }\end{array}$ & {$[20]$} \\
\hline & - & Berberine & Acute toxicity & Mouse & Induction of GU & [21] \\
\hline Cornus mas & Fruit/methanol extract & - & In vitro & - & Antioxidant & [22] \\
\hline $\begin{array}{l}\text { Cornus controversa, } \\
\text { C. macrophylla, and } \\
\text { C. walteri }\end{array}$ & Leaf/methanol extract & - & In vitro & - & Anti-H. pylori & {$[23]$} \\
\hline \multirow{3}{*}{ Cucurbita pepo } & Seed & Triterpenoids & In vitro & - & Antioxidant & {$[24]$} \\
\hline & $\begin{array}{l}\text { Ripe fruit pulp/aqueous } \\
\text { extract }\end{array}$ & - & $\begin{array}{l}\text { Aspirin-induced GU } \\
\text { and DU }\end{array}$ & Rat & $\begin{array}{l}\downarrow G U \text { and DU, } \uparrow \text { mucosal } \\
\text { thickness, and } \uparrow \text { alkaline } \\
\text { phosphatase enzyme in } \\
\text { stomach and duodenum } \\
\text { tissue }\end{array}$ & {$[25]$} \\
\hline & Seed & Triterpenoids & $\begin{array}{c}\text { Pyloric ligation-, water } \\
\text { immersion stress-, and } \\
\text { indomethacin-induced } \\
\text { GU }\end{array}$ & Rat & $\begin{array}{l}\downarrow G U \text { in all models, } \\
\downarrow \text { gastric secretion, and } \\
\downarrow \text { free and total acidity of } \\
\text { gastric juice }\end{array}$ & {$[24]$} \\
\hline \multirow{5}{*}{$\begin{array}{l}\text { Cydonia vulgaris } \\
\text { syn. C. oblonga }\end{array}$} & $\begin{array}{l}\text { Pulp, peel, and } \\
\text { seed/methanol extracts }\end{array}$ & - & In vitro & - & Antioxidant & {$[26]$} \\
\hline & Fruits/phenolic extract & - & In vitro & - & Antioxidant & [27] \\
\hline & Fruits/phenolic extract & - & Ethanol-induced GU & Rat & $\downarrow \mathrm{GU}$ & [27] \\
\hline & Seed/mucilage & - & $\begin{array}{l}\text { Toxin-induced skin } \\
\text { lesions }\end{array}$ & Rabbit & $\begin{array}{l}\text { Healing activity on } \\
\text { toxin-induced lesion }\end{array}$ & {$[28]$} \\
\hline & $\begin{array}{l}\text { Fruits juice } / 70 \% \text { ethanol } \\
\text { extract }\end{array}$ & - & In vitro & - & Anti-H. pylori activity & [29] \\
\hline \multirow[b]{2}{*}{ Malus domestica } & $\begin{array}{l}\text { Fruit juice and } \\
\text { flavonoids rich extract }\end{array}$ & & In vitro & - & Antioxidant & {$[30]$} \\
\hline & Fruit/methanol extract & $\begin{array}{l}\text { Catechin and } \\
\text { chlorogenic acid }\end{array}$ & In vitro & - & $\begin{array}{l}\downarrow \text { Gastric endothelial cell } \\
\text { injury caused by } \\
\text { xanthine-xanthine } \\
\text { oxidase and } \\
\text { indomethacin, } \\
\uparrow \text { antioxidant activity, } \\
\text { and } \downarrow \text { lipid peroxidation }\end{array}$ & {$[31]$} \\
\hline
\end{tabular}


TABle 2: Continued.

\begin{tabular}{|c|c|c|c|c|c|c|}
\hline Plant & Part/extract & Active constituent & Model & Species & Result & Reference \\
\hline & $\begin{array}{l}\text { Fruit } \\
\text { peel/polyphenol-rich } \\
\text { extract }\end{array}$ & - & In vitro & - & $\begin{array}{l}\text { Anti-H. pylori, } \\
\text { inhibition of } H \text {. } \\
\text { pylori-caused oxidant } \\
\text { and free radical } \\
\text { production, and } \\
\text { bacterial toxin } \\
\text { vacuolation and } \\
\text { adhesion to tissues }\end{array}$ & {$[32]$} \\
\hline & $\begin{array}{l}\text { Fruit/juice and } \\
\text { flavonoids extract }\end{array}$ & - & $\begin{array}{l}\mathrm{HCl} / \text { ethanol-induced } \\
\mathrm{GU}\end{array}$ & Rat & $\begin{array}{l}\downarrow \mathrm{GU}, \downarrow \text { MPO activity in } \\
\text { gastric tissue }\end{array}$ & {$[30]$} \\
\hline & Fruit/methanol extract & - & $\begin{array}{c}\text { Indomethacin-induced } \\
\text { PU }\end{array}$ & Rat & $\begin{array}{l}\downarrow P U, \downarrow \text { lipid peroxidation } \\
\text { and oxidative agents in } \\
\text { gastric tissue }\end{array}$ & {$[31]$} \\
\hline & $\begin{array}{l}\text { Peel/polyphenol-rich } \\
\text { extract }\end{array}$ & - & H. pylori infection & Mouse & $\begin{array}{l}\text { Suppression of } H . \\
\text { pylori-associated } \\
\text { gastritis, inflammation } \\
\text { and MDA levels in } \\
\text { gastric tissue }\end{array}$ & {$[33]$} \\
\hline & $\begin{array}{l}\text { Fruit/polyphenol } \\
\text { extract }\end{array}$ & - & $\begin{array}{c}\text { Aspirin-induced and } \\
\text { pylorus ligation-induced } \\
\text { GU }\end{array}$ & Rat & $\begin{array}{l}\downarrow G U \text { in both models, no } \\
\text { effect on gastric juice } \\
\text { secretion, inhibition of } \\
\text { aspirin-induced lipid } \\
\text { peroxidation, and } \\
\downarrow \text { COX } 2 \text { and HB-EGF } \\
\text { mRNA and protein over } \\
\text { expression }\end{array}$ & {$[34]$} \\
\hline & $\begin{array}{l}\text { Fruit/polyphenol } \\
\text { extract }\end{array}$ & - & Ethanol-induced GU & Rat & $\uparrow \mathrm{GU}$ & {$[35]$} \\
\hline \multirow{4}{*}{ Morus alba } & - & $\begin{array}{l}\text { Cyanidin-3- } \\
\text { Oglucoside }\end{array}$ & In vitro & - & $\begin{array}{l}\text { Improvement of } \\
\text { endothelial dysfunction }\end{array}$ & {$[36]$} \\
\hline & $\begin{array}{l}\text { Leaf/water and } 80 \% \\
\text { ethanol extracts }\end{array}$ & - & In vitro & - & $\begin{array}{l}\text { Anti-H. pylori, } \\
\text { antioxidant }\end{array}$ & {$[37]$} \\
\hline & $\begin{array}{l}\text { Fruit/ethyl acetate } \\
\text { soluble fraction }\end{array}$ & - & Stress-induced GU & Rat & $\begin{array}{l}\downarrow G U, \downarrow \text { oxidative stress in } \\
\text { tissue }\end{array}$ & {$[38]$} \\
\hline & Leaf/ethanol extract & - & Ethanol-induced GU & Rat & $\downarrow \mathrm{GU}$ & [35] \\
\hline \multirow{5}{*}{ Myristica fragrans } & Seed/methanol extract & - & In vitro & - & Anti-H. pylori & [39] \\
\hline & Aril of seed & $\begin{array}{l}\text { Dihydroguaiaretic } \\
\text { acid }\end{array}$ & In vitro & - & Anti-H. pylori & {$[40]$} \\
\hline & $\begin{array}{l}\text { Aril of seed/acetone } \\
\text { extract and its lignans } \\
\text { rich fraction }\end{array}$ & Lignans & In vitro & - & Antioxidant & {$[41]$} \\
\hline & Seed & - & $\begin{array}{l}\text { Carbachol-induced } \\
\text { gastric secretion }\end{array}$ & Rabbit & $\begin{array}{l}\downarrow \text { Gastric secretion, } \downarrow \text { free } \\
\text { and total acidity of } \\
\text { gastric juice }\end{array}$ & {$[42]$} \\
\hline & $\begin{array}{l}\text { Fruits in a polyherbal } \\
\text { formulation }\end{array}$ & - & $\begin{array}{c}\text { Pylorus } \\
\text { ligation-induced GU }\end{array}$ & Rat & $\begin{array}{l}\downarrow G U, \text { suppression of } \\
\text { gastric hypersecretion }\end{array}$ & {$[43]$} \\
\hline \multirow{2}{*}{$\begin{array}{l}\text { Myristica } \\
\text { malabarica }\end{array}$} & $\begin{array}{l}\text { Fruit rind/methanol } \\
\text { extract }\end{array}$ & - & $\begin{array}{l}\text { Indomethacin-induced } \\
\text { GU }\end{array}$ & Mouse & $\begin{array}{l}\uparrow \text { GU healing, } \uparrow \text { PGE2 } \\
\text { synthesis, and } \\
\uparrow \text { angiogenesis by } \\
\text { †pro-angiogenics: VEGF } \\
\text { and EGF }\end{array}$ & {$[44]$} \\
\hline & $\begin{array}{l}\text { Fruit rind/methanol } \\
\text { extract }\end{array}$ & - & $\begin{array}{l}\text { Indomethacin-induced } \\
\text { GU }\end{array}$ & Mouse & $\begin{array}{l}\uparrow \text { Ulcer healing, } \uparrow \text { eNOS } \\
\text { and } \downarrow \text { iNOS expressions, } \\
\text { and balance between } \\
\text { proinflammatory and } \\
\text { anti-inflammatory } \\
\text { cytokines }\end{array}$ & {$[45]$} \\
\hline
\end{tabular}


TABle 2: Continued.

\begin{tabular}{|c|c|c|c|c|c|c|}
\hline Plant & Part/extract & Active constituent & Model & Species & Result & Reference \\
\hline & $\begin{array}{l}\text { Fruits rind/methanol } \\
\text { extract }\end{array}$ & Procyanidins & $\begin{array}{l}\text { Indomethacin-induced } \\
\text { GU }\end{array}$ & Mouse & $\begin{array}{l}\uparrow G U \text { healing, } \uparrow \text { mucin } \\
\text { content, and } \downarrow \text { lipid } \\
\text { peroxidation and } \\
\uparrow \text { antioxidant activity of } \\
\text { gastric tissue }\end{array}$ & {$[46]$} \\
\hline $\begin{array}{l}\text { Myristica } \\
\text { andamanica }\end{array}$ & Leaf/methanol extract & - & Excision wound & Mouse & Wound healing activity & {$[47]$} \\
\hline \multirow{6}{*}{ Oryza sativa } & $\begin{array}{l}\text { Rice bran/methanol } \\
\text { extract }\end{array}$ & $\begin{array}{c}\text { Anthocyanins, } \\
\alpha \text {-tocopherol, and } \\
\gamma \text {-oryzanol }\end{array}$ & In vitro & - & Antioxidant & {$[48]$} \\
\hline & $\begin{array}{l}\text { Fluid from unpolished } \\
\text { and polished raw rice } \\
\text { and popularly cooked } \\
\text { Japanese rice }\end{array}$ & - & In vitro & - & Anti-H. pylori & {$[49]$} \\
\hline & Bran oil & - & Stress-induced GU & Rat & $\begin{array}{l}\downarrow G U, \downarrow \text { stress-induced } \\
\text { acid secretion, and } \\
\downarrow \text { basal and stimulated } \\
\text { acid secretion }\end{array}$ & {$[50]$} \\
\hline & Cooked fruit & - & Intestinal secretion assay & $\begin{array}{l}\text { Guinea } \\
\text { pigs }\end{array}$ & $\begin{array}{l}\downarrow \text { Intestinal secretion, } \\
\downarrow \text { response of intestinal } \\
\text { crypt cells to cAMP }\end{array}$ & {$[51]$} \\
\hline & $\begin{array}{l}\text { Stored rice bran oil, } \\
\text { fresh rice bran diet }\end{array}$ & - & Pylorus ligated ulcer & Rat & $\begin{array}{l}\uparrow \text { Ulcer by stored rice } \\
\text { bran oil, } \downarrow \text { ulcer by fresh } \\
\text { rice bran diet }\end{array}$ & {$[52]$} \\
\hline & Rice diet & - & Pylorus ligated ulcer & Rat & $\begin{array}{l}\downarrow \text { Gastric secretion, } \\
\downarrow \text { mucin activity, and } \\
\uparrow \text { ulcer severity }\end{array}$ & {$[53]$} \\
\hline \multirow{3}{*}{ Phoenix dactylifera } & $\begin{array}{l}\text { Fruit/methanol-water } \\
\text { extract }\end{array}$ & - & In vitro & - & Antioxidant & {$[54]$} \\
\hline & Seed/oil & - & In vitro & - & Antioxidant & {$[55]$} \\
\hline & $\begin{array}{l}\text { Fruit and pit/aqueous } \\
\text { and ethanol extracts }\end{array}$ & - & Ethanol-induced GU & Rat & $\begin{array}{l}\downarrow G U, \uparrow \text { gastric mucin, } \\
\downarrow \text { histamine in the gastric } \\
\text { mucosa, and } \downarrow \text { gastrin in } \\
\text { plasma }\end{array}$ & {$[56]$} \\
\hline \multirow{6}{*}{ Phyllanthus emblica } & Fruit & $\begin{array}{l}\text { Phenolic } \\
\text { compounds }\end{array}$ & In vitro & - & Antioxidant & {$[57]$} \\
\hline & Fruit/ethanol extract & - & In vitro & - & Anti-H. pylori & {$[58]$} \\
\hline & Fruit/ethanol extract & - & Excision wound & Rat & $\begin{array}{l}\text { } \text { Wound healing, } \\
\text { collagen function } \\
\text { improvement, and } \\
\text { †antioxidant enzymes: } \\
\text { SOD, GSH, and GPx }\end{array}$ & [59] \\
\hline & $\begin{array}{l}\text { Fruit/polar solvent } \\
\text { extract }\end{array}$ & - & Indomethacin-GU & Rat & $\begin{array}{l}\downarrow G U, \uparrow \text { antioxidant } \\
\text { activity, and } \\
\text { cytoprotective activity: } \\
\uparrow \text { mucus and hexosamine }\end{array}$ & {$[60]$} \\
\hline & $\begin{array}{l}\text { Fruit/gallic acid } \\
\text { enriched ethanol extract }\end{array}$ & - & $\begin{array}{l}\text { Indomethacin-induced } \\
\text { GU }\end{array}$ & Mouse & $\begin{array}{l}\uparrow G U \text { healing, } \uparrow \text { PGE2, } \\
\text { and } \uparrow \text { proangiogenesis } \\
\text { factors: VEGF, EGF, von } \\
\text { Willebrand Factor VIII, } \\
\text { and } \uparrow \text { eNOS/iNOS ratio }\end{array}$ & {$[61]$} \\
\hline & $\begin{array}{l}\text { Fruit/juice and } \\
\text { methanol extract }\end{array}$ & - & $\begin{array}{c}\text { Ethanol-, } \\
\text { indomethacin-, and } \\
\text { histamine-induced GU }\end{array}$ & Rat & $\begin{array}{l}\downarrow G U \text { in all models, } \\
\downarrow \text { intraluminal bleeding, } \\
\text { and } \uparrow \mathrm{GSH} \text { of mucus }\end{array}$ & {$[62]$} \\
\hline
\end{tabular}


TABLE 2: Continued.

\begin{tabular}{|c|c|c|c|c|c|c|}
\hline Plant & Part/extract & Active constituent & Model & Species & Result & Reference \\
\hline & $\begin{array}{l}\text { Gallic acid enriched } \\
\text { ethanol extract }\end{array}$ & & $\begin{array}{l}\text { Indomethacin-induced } \\
\text { GU }\end{array}$ & Mouse & $\begin{array}{l}\uparrow G U \text { healing, } \\
\text { proinflammatory and } \\
\text { anti-inflammatory } \\
\text { cytokines regulation, } \\
\text { antioxidant activity, and } \\
\downarrow l \text { lipid peroxidation }\end{array}$ & [63] \\
\hline \multirow{6}{*}{ Punica granatum } & $\begin{array}{l}\text { Fruit peel/aqueous } \\
\text { extract }\end{array}$ & - & In vitro & - & $\begin{array}{l}\downarrow \text { Acetylcholine-induced } \\
\text { contractions, } \\
\downarrow \text { spontaneous movement } \\
\text { of the isolated rat ileum }\end{array}$ & {$[64]$} \\
\hline & $\begin{array}{l}\text { Fruit peel/methanol } \\
\text { extract }\end{array}$ & - & In vitro & - & Anti-H. pylori & {$[65]$} \\
\hline & $\begin{array}{l}\text { Fruit peel/methanol } \\
\text { extract }\end{array}$ & - & Excision wound & $\begin{array}{l}\text { Guinea } \\
\text { pig }\end{array}$ & $\begin{array}{l}\uparrow \text { Wound healing, } \\
\uparrow \text { collagen, DNA, and } \\
\text { tissue proteins }\end{array}$ & {$[66]$} \\
\hline & $\begin{array}{l}\text { Fruit peel/aqueous } \\
\text { extract }\end{array}$ & - & Ethanol-induced GU & Rat & $\downarrow G U, \downarrow$ gastric acidity & {$[67]$} \\
\hline & $\begin{array}{l}\text { Fruit peel/methanol } \\
\text { extract }\end{array}$ & - & $\begin{array}{l}\text { Aspirin- and } \\
\text { ethanol-induced GU }\end{array}$ & Rat & $\begin{array}{l}\downarrow \text { GU in both models, } \\
\uparrow \text { catalase, } \uparrow \mathrm{GSH}, \uparrow \mathrm{GPx}, \\
\uparrow S O D, \text { and } \downarrow \text { lipid } \\
\text { peroxidation }\end{array}$ & {$[68]$} \\
\hline & Fruit & Tannins & $\begin{array}{l}\text { Water immersion } \\
\text { stress-, pylorus ligation-, } \\
\text { and intragastric absolute } \\
\text { ethanol-induced ulcer }\end{array}$ & Rat & $\begin{array}{l}\downarrow \text { Lipid peroxidation, } \\
\uparrow N O, \uparrow G P x, \uparrow S O D \text { in } \\
\text { gastric mucosa, and } \\
\uparrow \text { secretion of adherent } \\
\text { mucus and free mucus }\end{array}$ & [69] \\
\hline \multirow{3}{*}{ Rhus coriaria } & Fruit/ethanol extract & - & In vitro & - & Anti-H. pylori & {$[70]$} \\
\hline & Fruit/aqueous extract & Gallic acid & In vitro & - & $\begin{array}{l}\text { Antioxidant activity, } \\
\downarrow \text { oxidative stress, and } \\
\downarrow \text { lipid peroxidation in rat } \\
\text { isolated hepatocytes }\end{array}$ & {$[71]$} \\
\hline & Fruit/methanol extract & - & In vitro & - & $\begin{array}{l}\downarrow \text { lipid peroxidation, } \\
\text { Antioxidant activity }\end{array}$ & {$[72]$} \\
\hline \multirow{7}{*}{ Vitis vinifera } & Seed/various extract & - & In vitro & - & Antioxidant & [73] \\
\hline & $\begin{array}{l}\text { Fruit skin and } \\
\text { seed/various extract }\end{array}$ & - & In vitro & - & Anti-H. pylori & {$[74]$} \\
\hline & $\begin{array}{l}\text { Fruit/hydroalcoholic } \\
\text { extract }\end{array}$ & - & In vitro & - & Anti-H. pylori & {$[75]$} \\
\hline & Fruit juice & Resveratrol & In vitro & - & Anti-H. pylori & {$[76]$} \\
\hline & Fruit juice & Resveratrol & In vitro & - & $\begin{array}{l}\text { Anti-H. pylori, } \downarrow \text { ROS, } \\
\downarrow \text { inflammatory agents, } \\
\text { and improvement of } \\
\text { gastric mucosal cell } \\
\text { morphological changes } \\
\text { induced by H. pylori }\end{array}$ & {$[77]$} \\
\hline & $\begin{array}{l}\text { Seed/proanthocyanidin } \\
\text { extract }\end{array}$ & Resveratrol & $\begin{array}{l}\text { Acute and chronic } \\
\text { water-immersion } \\
\text { restraint stress-induced } \\
\text { gastric and intestinal } \\
\text { oxidative injury }\end{array}$ & Rat & $\begin{array}{l}\downarrow G U \text { and DU, } \downarrow \text { lipid } \\
\text { peroxidation, and } \\
\downarrow \text { gastric and duodenal } \\
\text { membrane } \\
\text { microviscosity }\end{array}$ & {$[78]$} \\
\hline & $\begin{array}{l}\text { Seed/low and high } \\
\text { flavanol content extract, } \\
\text { procyanidins extract }\end{array}$ & - & $\begin{array}{c}\text { Ethanol/HCl-induced } \\
\text { GU }\end{array}$ & Rat & $\begin{array}{l}\downarrow G U \text {, radical scavenging } \\
\text { activity, and } \\
\text { procyanidins binding } \\
\text { ability to stomach } \\
\text { surface protein which } \\
\text { result in } \uparrow \text { defense } \\
\text { activity of gastric } \\
\text { membrane }\end{array}$ & {$[79]$} \\
\hline
\end{tabular}


TABLE 2: Continued.

\begin{tabular}{|c|c|c|c|c|c|c|}
\hline Plant & Part/extract & Active constituent & Model & Species & Result & Reference \\
\hline & $\begin{array}{l}\text { Seed/proanthocyanidin } \\
\text { rich extract }\end{array}$ & - & $\begin{array}{l}\text { Aspirin- and } \\
\text { ethanol-induced GU }\end{array}$ & Rat & $\begin{array}{l}\downarrow \text { Ulcer in both models, } \\
\downarrow \text { lipid peroxidation more } \\
\text { than Vit E and Vit C }\end{array}$ & {$[80]$} \\
\hline & $\begin{array}{l}\text { Seed/proanthocyanidin } \\
\text { extract }\end{array}$ & - & Excision wound & Mouse & $\begin{array}{l}\uparrow \text { Wound healing, } \\
\text { †angiogenesis activity } \\
\text { and factor: VEGF, and } \\
\uparrow \text { antioxidant function of } \\
\text { tissue }\end{array}$ & [81] \\
\hline & - & Resveratrol & Aspirin-induced GU & Rat & $\begin{array}{l}2 \mathrm{mg} / \mathrm{Kg}: \uparrow \mathrm{GU} \text { healing, } \\
\downarrow \mathrm{MPO}, \uparrow \mathrm{COX} 1, \uparrow \mathrm{PGE} 2, \\
\uparrow \mathrm{eNOS} \text {, and } \\
\uparrow \text { angiogenesis; } 10 \mathrm{mg} / \mathrm{Kg}: \\
\text { ulcerogenic }\end{array}$ & {$[82]$} \\
\hline
\end{tabular}

cAMP: adenosine $3^{\prime}, 5^{\prime}$-cyclic monophosphate; COX: cyclooxygenase; DU: duodenal ulcer; EGF: epidermal growth factor; eNOS: endothelial NO synthase; GPx: glutathione peroxidase; GSH: glutathione; GU: gastric ulcer; H. pylori: Helicobacter pylori; HB-EGF: heparin-binding EGF-like growth factor; iNOS: inducible NO synthase; MDA: malondialdehyde; MPO: myeloperoxidase; NO: nitric oxide; PGE2: prostaglandin E2; PU: peptic ulcer; ROS: reactive oxygen species; SOD: superoxide dismutase; TNF- $\alpha$ : tumor necrosis factor-alpha; and VEGF: vascular EGF.

H. Pylori effects $[74,75]$. Proanthocyanidin-rich extract from seed protected against acute and chronic gastric and intestinal oxidative injury through inhibition of lipid peroxidation and membrane microviscosity in gastric and duodenal membrane [78]. It showed higher gastroprotective and antioxidant activity compared to vitamin E and C [80]. The seed also exhibited protective effect against gastric ulcer in rat. Antioxidant activity and strong ability of procyanidins to bind protein covering the stomach surface may be responsible for this protective affect [79]. This protein elevates defense activity of gastric membrane. The seed showed wound healing properties via enhancing angiogenesis and antioxidant activity $[81,83]$. Resveratrol, a high abundant polyphenol in red grape fruits, suppressed $H$. pylori growth, $H$. pylori-induced interleukin-8 secretion, reactive oxygen species generation, and morphological changes in human gastric epithelial cells $[76,77]$. Resveratrol in low dose $(2 \mathrm{mg} / \mathrm{Kg})$ demonstrated ulcer healing activity but in high dose $(10 \mathrm{mg} / \mathrm{Kg})$ was ulcerogenic. The mechanism of ulcer healing activity in low dose is attributed to inhibition of neutrophil aggregation, stimulation of COX1, PG $\mathrm{E}_{2}$, and eNOS, and improvement of angiogenesis [82].

\section{Discussion}

In TIM, a wide range of medicinal plants have been proposed for the treatment of different gastrointestinal disorders like inflammatory bowel disease, irritable bowel disease, hemorrhoids, and PU [84-87]. In this paper, all of edible fruits and spices claimed to be efficacious in the management of PU were collected from TIM sources, and any scientific evidence that prove their efficacy was retrieved from electronic databases. These remedies have shown their effectiveness on PU via several mechanisms of action including PG enhancement, modulation of inflammatory mediators, and antioxidant, anti $H$. pylori, wound healing, cytoprotective, and antisecretory activities. Some of the investigated fruits and spices like Myristica fragrans, Phyllantus embelica. Vitis vinifera, and Punica granatum have shown their beneficial effects in PU by affecting various associated mechanisms. According to published investigations, these fruits and spices seem to be more effective in the management of PU than the other ones. In contrast, for some of these fruits and spices including Morus species, Cornus mas, Rhus cariaria, and Phoenix dactylifera, just one or two studies on the efficacy and relevant mechanisms have been executed. Advanced scientific studies for evaluation of these herbs on PU and their possible mechanisms are suggested.

Despite many pieces of in vitro and in vivo evidence, no human study was found to confirm the effectiveness of investigated fruits and spices in PU. As shown in Table 1, the plants used in TIM for management of PU are from different families, and there is no exact relationship between the family of plants investigated and their efficacy. No potential side effects have been reported from these remedies. Studies on antiulcer activity of some of investigated fruits and spices have revealed controversial results. For example, stored rice bran oil has shown ulcerogenic activity. Whereas, fresh rice bran diet and rice diet have demonstrated anti-PU properties in animal models $[52,53]$. Fruit polyphenol extract of Malus domestica has ulcerogenic effect [27]. In contrast, fruit juice, flavonoids extract, and fruit methanol extract have shown gastroprotective activity in various animal models $[30,31,34]$. Despite different reports on protective activity of berberine, an active compound of Berberis vulgaris, against gastric ulcer $[19,20]$, there is a report about dosedependent gastric ulcer inducing activity of this compound [21]. Some of the investigated remedies have shown conflicting results in different doses. Resveratrol, a highly abundant polyphenol in Vitis vinifera fruit, in low dose demonstrated ulcer healing activity but in high dose was ulcerogenic [82].

Overall, there are various edible fruits and spices in TIM for the management of PU which their efficacy had confirmed through various in vitro and in vivo studies. Because of the 
lack of human studies, it is recommended to conduct clinical trials to prove their efficacy and obtain more conclusive results.

\section{Abbreviations}

COX: Cyclooxygenase

eNOS: Endothelial nitric oxide synthase

GSH: Glutathione

H. pylori: Helicobacter pylori

HB-EGF: Heparin-binding epidermal growth factor-like growth factor

NO: $\quad$ Nitric oxide

NOS: Nitric oxide synthase

NSAIDs: Non steroidal anti-inflammatory drugs

PU: $\quad$ Peptic ulcer

TIM: Traditional Iranian medicine

TNF- $\alpha$ : Tumor necrosis factor-alpha.

\section{Conflict of Interests}

The authors declare that they have no conflict of interests.

\section{References}

[1] S. Sumbul, M. A. Ahmad, M. Asif, and M. Akhtar, "Role of phenolic compounds in peptic ulcer: an overview," Journal of Pharmacy and Bioallied Sciences, vol. 3, no. 3, pp. 361-367, 2011.

[2] A. Soll and D. Graham, "Peptic ulcer disease," in Text Book of Gastroenterology, T. Yamada, Ed., pp. 936-941, Blackwell publication, Oxford, UK, 5th edition, 2009.

[3] G. N. J. Tytgat, "Etiopathogenetic principles and peptic ulcer disease classification," Digestive Diseases, vol. 29, no. 5, pp. 454458, 2011.

[4] J. M. B. Vítor and F. F. Vale, "Alternative therapies for Helicobacter pylori: probiotics and phytomedicine," FEMS Immunology and Medical Microbiology, vol. 63, no. 2, pp. 153-164, 2011.

[5] R. Rahimi, G. Amin, and M. R. Ardekani, "A review on Citrullus colocynthis Schrad.: from traditional Iranian medicine to modern phytotherapy," Journal of Alternative and Complementary Medicine, vol. 18, no. 6, pp. 551-554, 2012.

[6] R. Rahimi and M. R. Ardekani, "Medicinal properties of Foeniculum vulgare Mill. In traditional Iranian medicine and modern phytotherapy," Chinese Journal of Integrative Medicine, vol. 19, no. 1, pp. 73-79, 2013.

[7] M. R. S. Ardekani, R. Rahimi, B. Javadi, L. Abdi, and M. Khanavi, "Relationship between temperaments of medicinal plants and their major chemical compounds," Journal of Traditional Chinese Medicine, vol. 31, no. 1, pp. 27-31, 2011.

[8] M. H. Aghili, "Makhzan-al-Advia," R. Rahimi, M. R. ShamsArdekani, and F. Farjadmand, Eds., p. 700, Tehran University of Medical Sciences, Tehran, Iran, 2009, http://aqlibrary.org/ UserFiles/File/makhzan.pdf.

[9] Avicenna, The Cannon of Medicine, Sorush Publication, Tehran, Iran, 1983, translated from Arabic to Persian by Abdurrahman Sharaf-kandi.

[10] O. J. Houshia, M. AbuEid, O. Zaid, M. Zaid, and N. Aldaqqa, "Evaluation of the effectiveness of the acid-neutralizing contents of selected Palestinian folk medicinal herbs," Advances in Pure and Applied Chemistry, vol. 1, no. 4, pp. 77-79, 2012.
[11] A. J. Sfahlan, A. Mahmoodzadeh, A. Hasanzadeh, R. Heidari, and R. Jamei, "Antioxidants and antiradicals in almond hull and shell (Amygdalus communis L.) as a function of genotype," Food Chemistry, vol. 115, no. 2, pp. 529-533, 2009.

[12] R. Amarowicz, A. Troszyńska, and F. Shahidi, "Antioxidant activity of almond seed extract and its fractions," Journal of Food Lipids, vol. 12, no. 4, pp. 344-358, 2005.

[13] M. Bayat, R. Tarighat, and A. Khoshvaghti, "The effect of bitter almond oil on open skin wound healing: a tensiometrical study," Iranian Journal of Pharmaceutical Research, vol. 3, supplement 2, article 162, p. 56, 2004, Poster Presentations.

[14] F. Nabavizadeh, A. M. Alizadeh, Z. Sadroleslami, and S. Adeli, "Gastroprotective effects of amygdalin on experimental gastric ulcer: role of NO and TNF- $\alpha$," Journal of Medicinal Plants Research, vol. 5, no. 14, pp. 3122-3127, 2011.

[15] G. Motalleb, P. Hanachi, S. H. Kua, O. Fauziah, and R. Asmah, "Evaluation of phenolic content and total antioxidant activity in Berberis vulgaris fruit extract," Journal of Biological Science, vol. 5, pp. 648-653, 2005.

[16] Z.-W. Suo, G. Hu, and H.-Q. Duan, "Effect of berberine on nitric oxide release of rat intestinal mucous microvascular endothelial cells," Zhongguo Zhong Xi Yi Jie He Za Zhi, vol. 27, no. 9, pp. 832834, 2007.

[17] J. G. Chung, L. T. Wu, S. H. Chang et al., "Inhibitory actions of berberine on growth and arylamine $\mathrm{N}$-acetyltransferase activity in strains of Helicobacter pylori from peptic ulcer patients," International Journal of Toxicology, vol. 18, no. 1, pp. 35-40, 1998.

[18] A. Yazdani, S. L. Poorbaghi, H. Habibi, S. Nazifi, F. Rahmani Far, and M. Sepehrimanesh, "Dietary Berberis vulgaris extract enhances intestinal mucosa morphology in the broiler chicken (Gallus gallus)," Comparative Clinical Pathology, vol. 22, no. 4, pp. 611-615, 2012.

[19] L.-R. Pan, Q. Tang, Q. Fu, B.-R. Hu, J.-Z. Xiang, and J.-Q. Qian, "Roles of nitric oxide in protective effect of berberine in ethanolinduced gastric ulcer mice," Acta Pharmacologica Sinica, vol. 26, no. 11, pp. 1334-1338, 2005.

[20] Y. Watanabe-Fukuda, M. Yamamoto, N. Miura et al., "Orengedokuto and berberine improve indomethacin-induced small intestinal injury via adenosine," Journal of Gastroenterology, vol. 44, no. 5, pp. 380-389, 2009.

[21] E. Yeşilada and E. Küpeli, "Berberis crataegina DC. root exhibits potent anti-inflammatory, analgesic and febrifuge effects in mice and rats," Journal of Ethnopharmacology, vol. 79, no. 2, pp. 237-248, 2002.

[22] H. Hassanpour, H. Yousef, H. Jafar, and A. Mohammad, "Antioxidant capacity and phytochemical properties of cornelian cherry (Cornus mas L.) genotypes in Iran," Scientia Horticulturae, vol. 129, no. 3, pp. 459-463, 2011.

[23] H. K. Lee, H. B. Lee, C. S. Kim, and Y. J. Ahn, "Anti-Helicobacter pylori activity of methanol extracts from Korean native plant species in Jeju island," Agricaltural Chemistry and Biotechnology, vol. 47, no. 2, pp. 91-96, 2004.

[24] N. S. Gill and M. Bali, "Isolation of anti ulcer cucurbitane type triterpenoid from the seeds of cucurbita pepo," Research Journal of Phytochemistry, vol. 5, no. 2, pp. 70-79, 2011.

[25] S. Sarkar and D. Guha, "Effect of ripe fruit pulp extract of Cucurbita pepo Linn. in aspirin induced gastric and duodenal ulcer in rats," Indian Journal of Experimental Biology, vol. 46, no. 9, pp. 639-645, 2008.

[26] B. M. Silva, P. B. Andrade, P. Valentão, F. Ferreres, R. M. Seabra, and M. A. Ferreira, "Quince (Cydonia oblonga Miller) fruit 
(pulp, peel, and seed) and jam: antioxidant activity," Journal of Agricultural and Food Chemistry, vol. 52, no. 15, pp. 4705-4712, 2004.

[27] Y. Hamauzu, T. Inno, C. Kume, M. Irie, and K. Hiramatsu, "Antioxidant and antiulcerative properties of phenolics from Chinese quince, quince, and apple fruits," Journal of Agricultural and Food Chemistry, vol. 54, no. 3, pp. 765-772, 2006.

[28] A. A. Hemmati, H. Kalantari, A. Jalali, S. Rezai, and H. H. Zadeh, "Healing effect of quince seed mucilage on T-2 toxininduced dermal toxicity in rabbit," Experimental and Toxicologic Pathology, vol. 64, no. 3, pp. 181-186, 2012.

[29] A. Babarikina, V. Nikolajeva, and D. Babarykin, "AntiHelicobacter activity of certain food plant extracts and juices and their composition in vitro," Food and Nutrition Sciences, vol. 2, pp. 868-877, 2011.

[30] Y. Hamauzu, M. Irie, M. Kondo, and T. Fujita, "Antiulcerative properties of crude polyphenols and juice of apple, and Chinese quince extracts," Food Chemistry, vol. 108, no. 2, pp. 488-495, 2008.

[31] G. Graziani, G. D’Argenio, C. Tuccillo et al., "Apple polyphenol extracts prevent damage to human gastric epithelial cells in vitro and to rat gastric mucosa in vivo," Gut, vol. 54, no. 2, pp. 193200, 2005.

[32] E. Pastene, H. Speisky, M. Troncoso, J. Alarcón, and G. Figueroa, "In vitro inhibitory effect of apple peel extract on the growth of Helicobacter pylori and respiratory burst induced on human neutrophils," Journal of Agricultural and Food Chemistry, vol. 57, no. 17, pp. 7743-7749, 2009.

[33] E. Pastene, H. Speisky, A. García, J. Moreno, M. Troncoso, and G. Figueroa, "In vitro and in vivo effects of apple peel polyphenols against Helicobacter pylori," Journal of Agricultural and Food Chemistry, vol. 58, no. 12, pp. 7172-7179, 2010.

[34] G. D’Argenio, G. Mazzone, C. Tuccillo et al., "Apple polyphenol extracts prevent aspirin-induced damage to the rat gastric mucosa," British Journal of Nutrition, vol. 100, no. 6, pp. 12281236, 2008.

[35] M. A. Abdulla, H. M. Ali, K. A.-A. Ahmed, S. M. Noor, and S. Ismail, "Evaluation of the anti-ulcer activities of Morus alba extracts in experimentally-induced gastric ulcer in rats," Biomedical Research, vol. 20, no. 1, pp. 35-39, 2009.

[36] I. Serraino, L. Dugo, P. Dugo et al., "Protective effects of cyanidin-3-O-glucoside from blackberry extract against peroxynitrite-induced endothelial dysfunction and vascular failure," Life Sciences, vol. 73, no. 9, pp. 1097-1114, 2003.

[37] Y. J. Cho, I. S. Ju, B. O. Kim et al., "The antimicrobial activity against Helicobacter pylori and antioxidant effect from the extracts of mulberry leaves (Morus alba L.)," Journal of the Korean Society for Applied Biological Chemistry, vol. 50, no. 4, pp. 334-343, 2007.

[38] V. S. Nade and A. V. Yadav, "Anti-stress effect of ethyl acetate soluble fraction of Morus alba in chronic restraint stress," Pharmaceutical Biology, vol. 48, no. 9, pp. 1038-1046, 2010.

[39] G. B. Mahady, S. L. Pendland, A. Stoia et al., "In vitro susceptibility of Helicobacter pylori to botanical extracts used traditionally for the treatment of gastrointestinal disorders," Phytotherapy Research, vol. 19, no. 11, pp. 988-991, 2005.

[40] S. Bhamarapravati, S. Juthapruth, W. Mahachai, and G. Mahady, "Antibacterial activity of Boesenbergia rotunda (L.) mansf. and Myristica fragrans houtt. against Helicobacter pylori," Songklanakarin Journal of Science and Technology, vol. 28, no. 1, pp. 157-163, 2006.
[41] S. Chatterjee, Z. Niaz, S. Gautam, S. Adhikari, P. S. Variyar, and A. Sharma, "Antioxidant activity of some phenolic constituents from green pepper (Piper nigrum L.) and fresh nutmeg mace (Myristica fragrans)," Food Chemistry, vol. 101, no. 2, pp. 515523, 2007.

[42] M. Jan, F. Faqir, H. Hamida, and M. A. Mughal, "Comparison of effects of extract of Myristica fragrans and verapamil on the volume and acidity of carbachol induced gastric secretion in fasting rabbits," Journal of Ayub Medical College, Abbottabad, vol. 17, no. 2, pp. 69-71, 2005.

[43] P. Bafna and S. Bodhankar, "Gastrointestinal effects of Mebarid, an ayurvedic formulation, in experimental animals," Journal of Ethnopharmacology, vol. 86, no. 2-3, pp. 173-176, 2003.

[44] B. Maity, D. Banerjee, S. K. Bandyopadhyay, and S. Chattopadhyay, "Myristica malabarica heals stomach ulceration by increasing prostaglandin synthesis and angiogenesis," Planta Medica, vol. 74, no. 15, pp. 1774-1778, 2008.

[45] B. Maity, D. Banerjee, S. K. Bandyopadhyay, and S. Chattopadhyay, "Regulation of arginase/nitric oxide synthesis axis via cytokine balance contributes to the healing action of malabaricone $\mathrm{B}$ against indomethacin-induced gastric ulceration in mice," International Immunopharmacology, vol. 9, no. 4, pp. 491498, 2009.

[46] D. Banerjee, B. Maity, A. K. Bauri, S. K. Bandyopadhyay, and S. Chattopadhyay, "Gastroprotective properties of Myristica malabarica against indometacin-induced stomach ulceration: a mechanistic exploration," Journal of Pharmacy and Pharmacology, vol. 59, no. 11, pp. 1555-1565, 2007.

[47] K. D. Arunachalam and S. Subhashini, "Preliminary phytochemical investigation and wound healing activity of Myristica andamanica leaves in Swiss albino mice," Journal of Medicinal Plant Research, vol. 5, no. 7, pp. 1095-1106, 2011.

[48] T. Laokuldilok, C. F. Shoemaker, S. Jongkaewwattana, and V. Tulyathan, "Antioxidants and antioxidant activity of several pigmented rice brans," Journal of Agricultural and Food Chemistry, vol. 59, no. 1, pp. 193-199, 2011.

[49] Y. Kawakami, K. Oana, M. Hayama et al., "In vitro bactericidal activities of Japanese rice-fluid against Helicobacter pylori strains," International Journal of Medical Sciences, vol. 3, no. 3, pp. 112-116, 2006.

[50] J. M. Lloris, B. Bolant, L. Gimeno et al., "Gastric juice and analysis of basal and stimulated secretion following treatment with rice-bran oil," Research Communications in Chemical Pathology and Pharmacology, vol. 74, no. 2, pp. 245-248, 1991.

[51] R. J. Macleod, H. P. J. Bennett, and J. R. Hamilton, "Inhibition of intestinal secretion by rice," The Lancet, vol. 346, no. 8967, pp. 90-92, 1995.

[52] A. P. Jayaraj, F. I. Tovey, and C. G. Clark, "The ulcerogenic and protective action of rice and rice fractions in experimental peptic ulceration," Clinical Science, vol. 72, no. 4, pp. 463-466, 1987.

[53] K. P. Singh and R. H. Singh, "Effect of rice, wheat and arahara diet on peptic ulceration: a clinical (retrospect) and experimental study," Ancient Science of Life, vol. 8, no. 3-4, pp. 250-257, 1989.

[54] F. Biglari, A. F. M. AlKarkhi, and A. M. Easa, "Antioxidant activity and phenolic content of various date palm (Phoenix dactylifera) fruits from Iran," Food Chemistry, vol. 107, no. 4, pp. 1636-1641, 2008.

[55] M. Biglar, M. Khanavi, M. Hajimahmoodi et al., "Tocopherol content and fatty acid profile of different Iranian date seed oils," 
Iranian Journal of Pharmaceutical Research, vol. 11, no. 3, pp. 873-878, 2012.

[56] A. A. Al-Qarawi, H. Abdel-Rahman, B. H. Ali, H. M. Mousa, and S. A. El-Mougy, "The ameliorative effect of dates (Phoenix dactylifera L.) on ethanol-induced gastric ulcer in rats," Journal of Ethnopharmacology, vol. 98, no. 3, pp. 313-317, 2005.

[57] W. Luo, M. Zhao, B. Yang, J. Ren, G. Shen, and G. Rao, "Antioxidant and antiproliferative capacities of phenolics purified from Phyllanthus emblica L. fruit," Food Chemistry, vol. 126, no. 1, pp. 277-282, 2011.

[58] S. Mehrotra, R. Jamwal, R. Shyam et al., "Anti-Helicobacter pylori and antioxidant properties of Emblica officinalis pulp extract: a potential source for therapeutic use against gastric ulcer," Journal of Medicinal Plant Research, vol. 5, no. 12, pp. 2577-2583, 2011.

[59] M. Sumitra, P. Manikandan, V. S. Gayathri, P. Mahendran, and L. Suguna, "Emblica officinalis exerts wound healing action through up-regulation of collagen and extracellular signalregulated kinases (ERK1/2)," Wound Repair and Regeneration, vol. 17, no. 1, pp. 99-107, 2009.

[60] S. K. Bandyopadhyay, S. C. Pakrashi, and A. Pakrashi, "The role of antioxidant activity of Phyllanthus emblica fruits on prevention from indomethacin induced gastric ulcer," Journal of Ethnopharmacology, vol. 70, no. 2, pp. 171-176, 2000.

[61] A. Chatterjee, S. Chatterjee, A. Biswas, S. Bhattacharya, S. Chattopadhyay, and S. K. Bandyopadhyay, "Gallic acid enriched fraction of Phyllanthus emblica potentiates indomethacininduced gastric ulcer healing via e-NOS-dependent pathway," Evidence-Based Complementary and Alternative Medicine, vol. 2012, Article ID 487380, 13 pages, 2012.

[62] N. V. Rajeshkumar, M. Therese, and R. Kuttan, "Emblica officinalis fruits afford protection against experimental gastric ulcers in rats," Pharmaceutical Biology, vol. 39, no. 5, pp. 375380, 2001

[63] S. K. Bandyopadhyay, A. Chatterjee, and S. Chattopadhyay, "Biphasic effect of Phyllanthus emblica L. extract on NSAIDinduced ulcer: an antioxidative trail weaved with immunomodulatory effect," Evidence-Based Complementary and Alternative Medicine, vol. 2011, Article ID 146808, 13 pages, 2011.

[64] E. Y. Qnais, A. S. Elokda, Y. Y. A. Ghalyun, and F. A. Abdulla, "Antidiarrheal activity of the aqueous extract of Punica granatum (pomegranate) peels," Pharmaceutical Biology, vol. 45, no. 9, pp. 715-720, 2007.

[65] M. Hajimahmoodi, M. Shams-Ardakani, P. Saniee et al., "In vitro antibacterial activity of some Iranian medicinal plant extracts against Helicobacter pylori," Natural Product Research, vol. 25, no. 11, pp. 1059-1066, 2011.

[66] E. A. Hayouni, K. Miled, S. Boubaker et al., "Hydroalcoholic extract based-ointment from Punica granatum L. peels with enhanced in vivo healing potential on dermal wounds," Phytomedicine, vol. 18, no. 11, pp. 976-984, 2011.

[67] K. Gharzouli, S. Khennouf, S. Amira, and A. Gharzouli, "Effects of aqueous extracts from Quercus ilex L. root bark, Punica granatum L. fruit peel and Artemisia herba-alba Asso leaves on ethanol-induced gastric damage in rats," Phytotherapy Research, vol. 13, no. 1, pp. 42-45, 1999.

[68] K. B. Ajaikumar, M. Asheef, B. H. Babu, and J. Padikkala, "The inhibition of gastric mucosal injury by Punica granatum L. (pomegranate) methanolic extract," Journal of Ethnopharmacology, vol. 96, no. 1-2, pp. 171-176, 2005.
[69] S. Lai, Q. Zhou, Y. Zhang, J. Shang, and T. Yu, "Effects of Pomegranate tannins on experimental gastric damages," Zhongguo Zhong Yao Za Zhi, vol. 34, no. 10, pp. 1290-1294, 2009.

[70] Y. Motaharinia, M. S. Hazhir, M. A. Rezaee et al., "Comparison of in vitro antimicrobial effect of ethanol extracts of Satureja khuzestanica, Rhus coriaria, and Ocimum basilicum L. on Helicobacter pylori," Journal of Medicinal Plants Research, vol. 6, no. 21, pp. 3749-3753, 2012.

[71] J. Pourahmad, M. R. Eskandari, R. Shakibaei, and M. Kamalinejad, "A search for hepatoprotective activity of aqueous extract of Rhus coriaria L. against oxidative stress cytotoxicity," Food and Chemical Toxicology, vol. 48, no. 3, pp. 854-858, 2010.

[72] F. Candan and A. Sökmen, "Effects of Rhus coriaria L. (Anacardiaceae) on lipid peroxidation and free radical scavenging activity," Phytotherapy Research, vol. 18, no. 1, pp. 84-86, 2004.

[73] G. K. Jayaprakasha, R. P. Singh, and K. K. Sakariah, "Antioxidant activity of grape seed (Vitis vinifera) extracts on peroxidation models in vitro," Food Chemistry, vol. 73, no. 3, pp. 285-290, 2001.

[74] J. C. Brown, G. Huang, V. Haley-Zitlin, and X. Jiang, "Antibacterial effects of grape extracts on Helicobacter pylori," Applied and Environmental Microbiology, vol. 75, no. 3, pp. 848-852, 2009.

[75] S. Martini, C. D’Addario, D. Braconi et al., "Antibacterial activity of grape extracts on cagA-positive and -negative Helicobacter pylori clinical isolates," Journal of Chemotherapy, vol. 21, no. 5, pp. 507-513, 2009.

[76] G. B. Mahady, S. L. Pendland, and L. R. Chadwick, "Resveratrol and red wine extracts inhibit the growth of CagA+ strains of Helicobacter pylori in vitro," American Journal of Gastroenterology, vol. 98, no. 6, pp. 1440-1441, 2003.

[77] S. F. H. Zaidi, K. Ahmed, T. Yamamoto et al., "Effect of resveratrol on Helicobacter pylori-induced interleukin- 8 secretion, reactive oxygen species generation and morphological changes in human gastric epithelial cells," Biological and Pharmaceutical Bulletin, vol. 32, no. 11, pp. 1931-1935, 2009.

[78] M. Bagchi, M. Milnes, C. Williams et al., "Acute and chronic stress-induced oxidative gastrointestinal injury in rats, and the protective ability of a novel grape seed proanthocyanidin extract," Nutrition Research, vol. 19, no. 8, pp. 1189-1199, 1999.

[79] M. Saito, H. Hosoyama, T. Ariga, S. Kataoka, and N. Yamaji, "Antiulcer activity of grape seed extract and procyanidins," Journal of Agricultural and Food Chemistry, vol. 46, no. 4, pp. 1460-1464, 1998.

[80] V. M. Cuevas, Y. R. Calzado, Y. P. Guerra et al., "Effects of grape seed extract, vitamin $C$, and vitamin e on ethanol- and aspirininduced ulcers," Advances in Pharmacological Sciences, vol. 2011, Article ID 740687, 6 pages, 2011.

[81] S. Khanna, M. Venojarvi, S. Roy et al., "Dermal wound healing properties of redox-active grape seed proanthocyanidins," Free Radical Biology and Medicine, vol. 33, no. 8, pp. 1089-1096, 2002.

[82] A. Dey, P. Guha, S. Chattopadhyay, and S. K. Bandyopadhyay, "Biphasic activity of resveratrol on indomethacin-induced gastric ulcers," Biochemical and Biophysical Research Communications, vol. 381, no. 1, pp. 90-95, 2009.

[83] L. Paulo, M. Oleastro, E. Gallardo, J. A. Queiroz, and F. Domingues, "Anti-Helicobacter pylori and urease inhibitory activities of resveratrol and red wine," Food Research International, vol. 44, no. 4, pp. 964-969, 2011.

[84] R. Rahimi, M. R. Shams-Ardekani, and M. Abdollahi, "A review of the efficacy of traditional Iranian medicine for inflammatory bowel disease," World Journal of Gastroenterology, vol. 16, no. 36, pp. 4504-4514, 2010. 
[85] R. Rahimi and M. Abdollahi, "Herbal medicines for the management of irritable bowel syndrome: a comprehensive review," World Journal of Gastroenterology, vol. 18, no. 7, pp. 589-600, 2012.

[86] R. Rahimi and M. Abdollahi, "Evidence-based review of medicinal plants used for the treatment of hemorrhoids," International Journal of Pharmacology, vol. 9, pp. 1-11, 2013.

[87] M. H. Farzaei, M. Khazaei, Z. Abasabadi, M. Feyzmahdavi, and G. R. Mohseni, "Protective effect of Trogopogon graminifolius DC. against ethanol induced gastric ulcer in rat," Iranian Red Crescent Medical Journal. In press. 


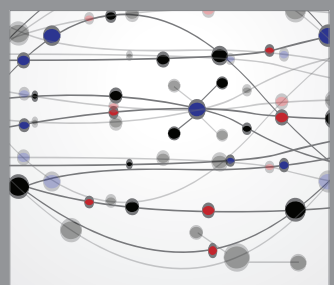

The Scientific World Journal
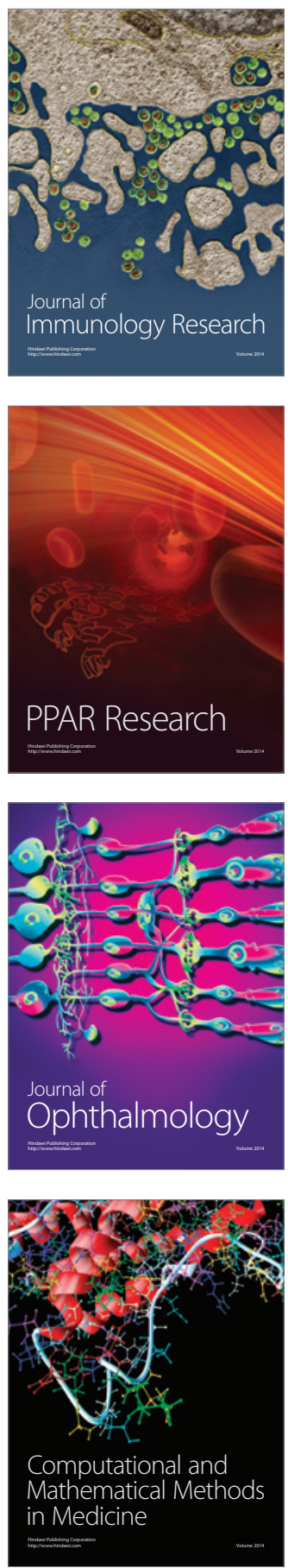

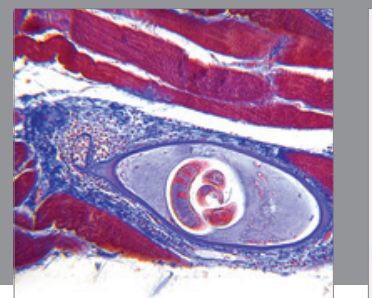

Gastroenterology

Research and Practice
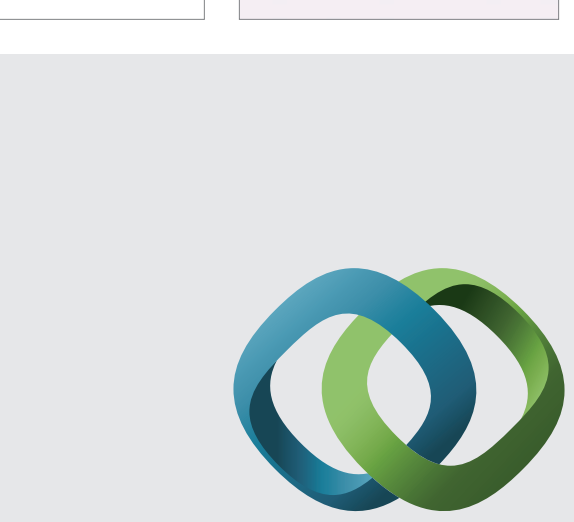

\section{Hindawi}

Submit your manuscripts at

http://www.hindawi.com
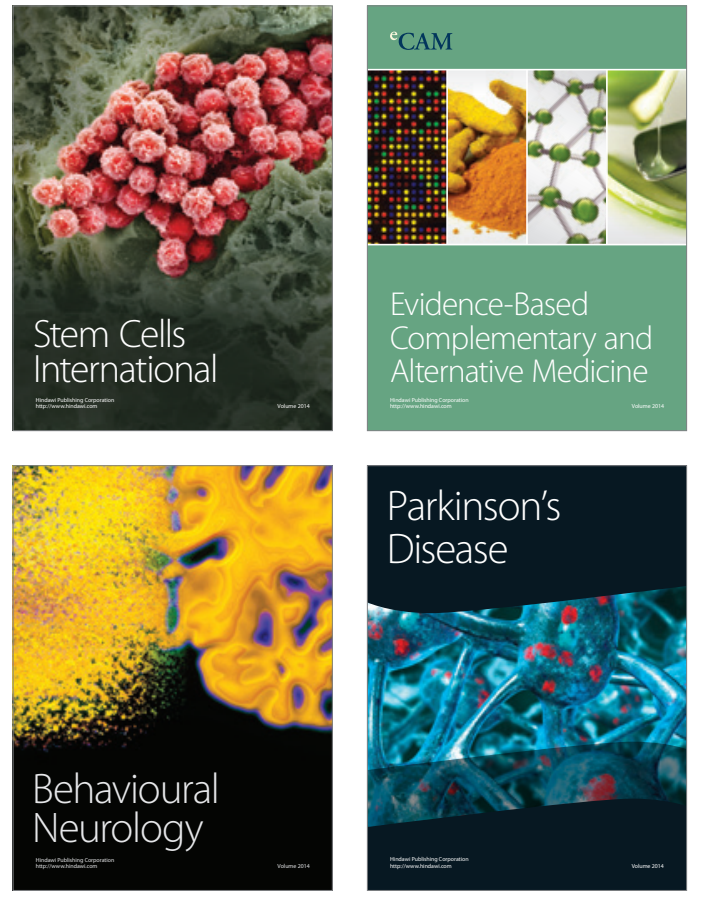
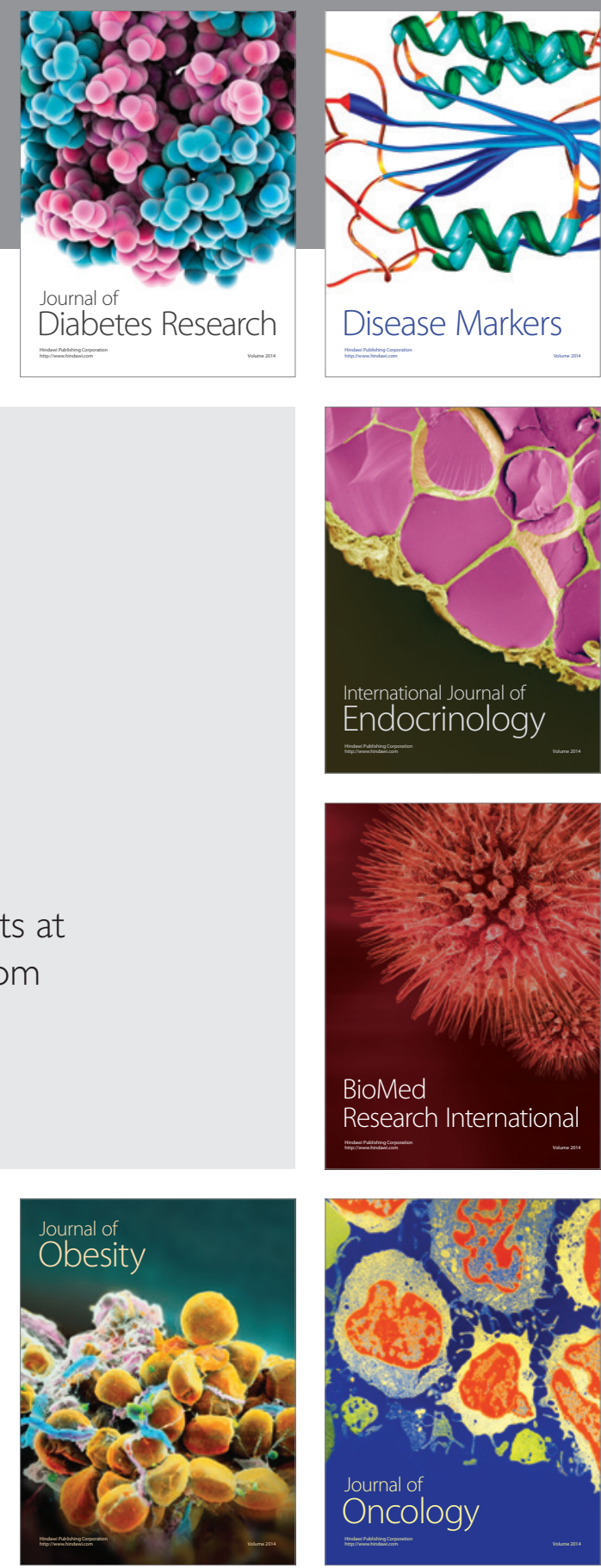

Disease Markers
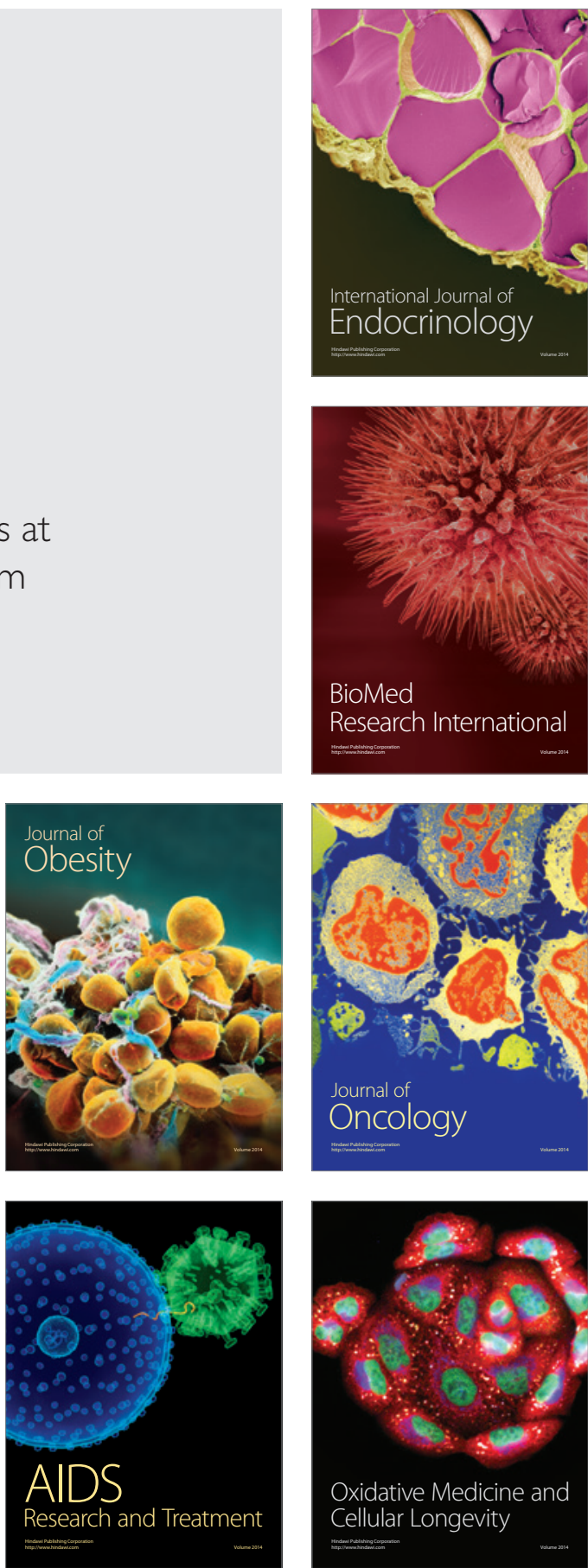